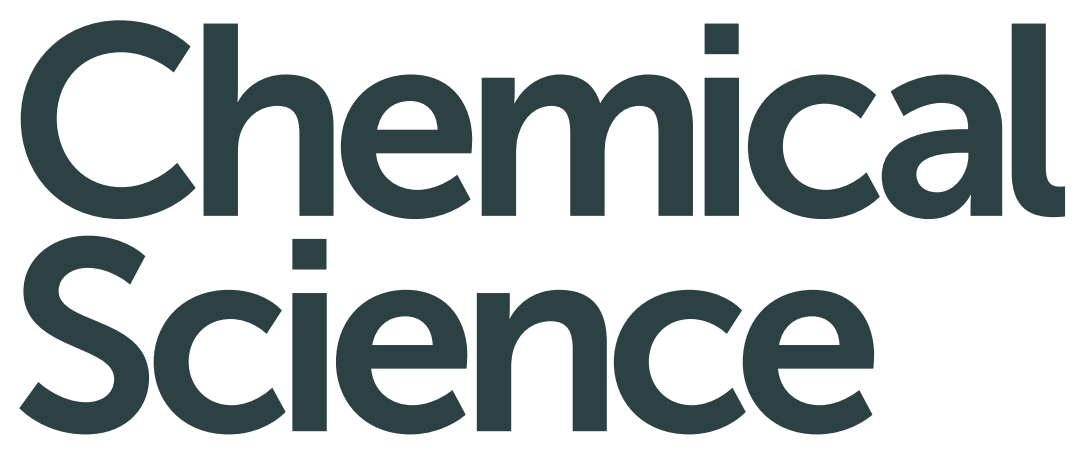

rsc.li/chemical-science

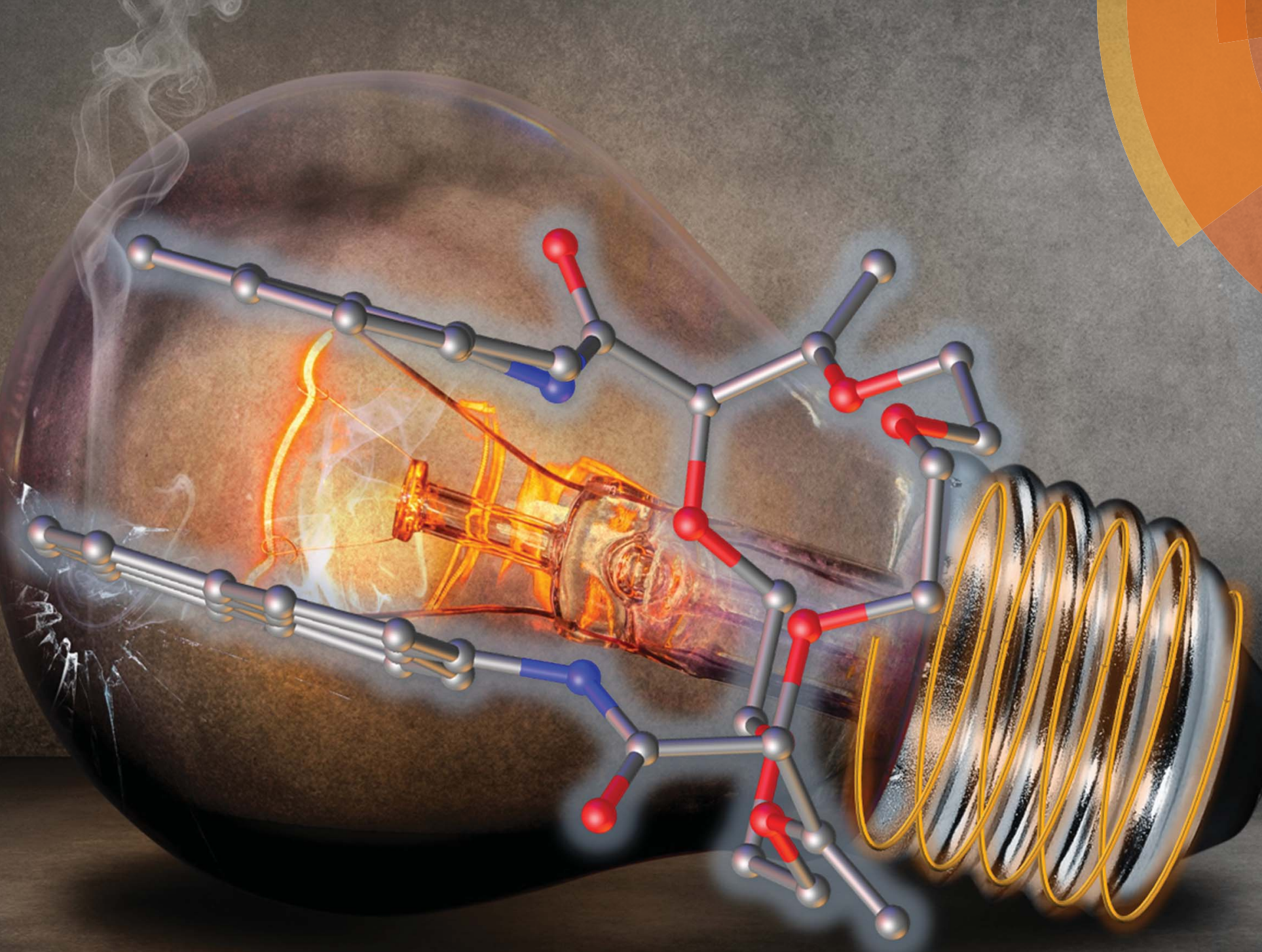

ISSN 2041-6539

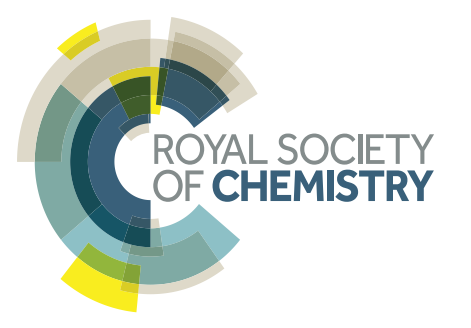


Check for updates

Cite this: Chem. Sci., 2018, 9, 7043

๑ All publication charges for this article have been paid for by the Royal Society of Chemistry

Received 3rd July 2018

Accepted 30th July 2018

DOI: $10.1039 / \mathrm{c} 8 \mathrm{sc} 02935 \mathrm{k}$

rsc.li/chemical-science

\title{
Combined reversible switching of ECD and quenching of CPL with chiral fluorescent macrocycles $\uparrow$
}

\author{
Alexandre Homberg, (D) $\S^{a}$ Elodie Brun, (D) $\S^{a}$ Francesco Zinna, (D) $\S^{a}$ Simon Pascal, (D) $\ddagger^{a}$ \\ Marcin Górecki, ${ }^{\mathrm{b}}$ Luc Monnier, ${ }^{\mathrm{a}}$ Céline Besnard, (D) ${ }^{\mathrm{c}}$ Gennaro Pescitelli, (D) ${ }^{\mathrm{b}}$ \\ Lorenzo Di Bari (D) ${ }^{b}$ and Jérôme Lacour (D) *a
}

\begin{abstract}
Straightforward synthesis and resolution of a series of chiral fluorescent macrocycles are presented, together with their electronic circular dichroism (ECD), strong excimer fluorescence (EF, $\lambda 300$ to 650 $\mathrm{nm}$ ) and allied highly circularly polarized luminescence (CPL, $g_{\text {lum }}$ up to $1.7 \times 10^{-2}$ ). The ECD, EF and $\mathrm{CPL}$ responses are strongly affected by the presence of metal ions $\left(\mathrm{Na}^{+}, \mathrm{Ba}^{2+}\right)$ thanks to deep conformational changes. While ECD signals can be almost completely reversibly inverted upon the complexation/decomplexation of metal ions in a typical binary response, CPL signals are reversibly quenched concomitantly. The designed macrocycles display thus a remarkable combination of both + /ECD and on/off CPL reversible switching.
\end{abstract}

\section{Introduction}

Tuning the chiroptical properties of molecular or supramolecular systems is of pivotal importance for applications in material science. ${ }^{1}$ Key examples have been reported for the development of switchable memories $^{2}$ and molecular machines, ${ }^{3}$ polarized light emitting devices, ${ }^{4}$ chiral transistors $^{4 a, 5}$ and chiral spin filters. ${ }^{6}$ Molecules of particular interest are those able to switch their optical properties in response to an external stimulus in a reversible fashion. ${ }^{7}$ Interactions with circularly polarized light, either in absorption or in emission, can then give unambiguous information about molecular states. Reversible electronic circular dichroism (ECD) switches have been reported. They are usually based on metallated helicenes,${ }^{7 a, 8}$ gels, ${ }^{9}$ polymers,${ }^{10}$ lanthanide complexes ${ }^{11}$ or supramolecular assemblies ${ }^{12}$ and can be triggered by light, ${ }^{3,13}$

${ }^{a}$ Department of Organic Chemistry, University of Geneva, Quai Ernest Ansermet 30 , 1211 Geneva 4, Switzerland. E-mail: Jerome.lacour@unige.ch

${ }^{b}$ Dipartimento di Chimica e Chimica Industriale, Università di Pisa, Via Moruzzi 13, 56124 Pisa, Italy

'Laboratoire de Cristallographie, University of Geneva, Quai Ernest Ansermet 24, 1211 Geneva 4, Switzerland

$\dagger$ Electronic supplementary information (ESI) available: Experimental conditions, full characterizations, ${ }^{1} \mathrm{H}$ NMR and ${ }^{13} \mathrm{C}$ NMR spectra of all new compounds (PDF); CSP-HPLC traces; UV-Vis, ECD, fluorescence and CPL spectra; computational details. CCDC 1045592 and 1045593. For ESI and crystallographic data in CIF or other electronic format see DOI: $10.1039 / \mathrm{c} 8 \mathrm{sc} 02935 \mathrm{k}$

$\S$ These authors contributed equally to this work.

‡ Current address: CINaM, Aix-Marseille Université, UMR CNRS 7325, Campus de Luminy, case 913, 13288 Marseille Cedex 9, France. electrochemical processes, ${ }^{14}$ thermal or mechanic stress, ${ }^{15} \mathrm{pH}$ variations, ${ }^{16}$ ions coordination ${ }^{17}$ or concentration. ${ }^{18}$

In addition to ECD, circular polarization of emitted light can be exploited as a selective read-out. Reversible circularly polarized luminescence (CPL) switches remain however rare, ${ }^{7 a, 8 a, 16}$ in particular for purely organic derivatives. For instance (Fig. 1), the groups of Longhi and Cuerva designed a macrocyclic $o$-oligo(phenylene)ethynylene on/off CPL switch by reversible $\mathrm{Ag}^{+}$ coordination. ${ }^{17 b, 19}$ Similarly, Nakashima, Kawai and coworkers reported a reversible on/off CPL switch of a bispyrene-bearing

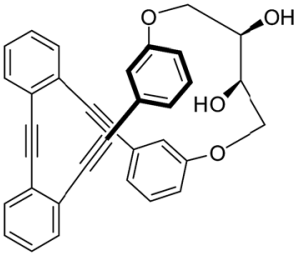

Longhi and Cuerva 2016

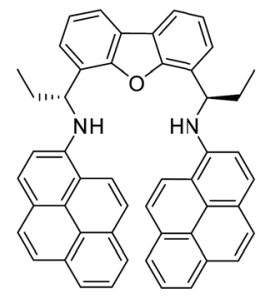

Ito, Imai and Asami 2017
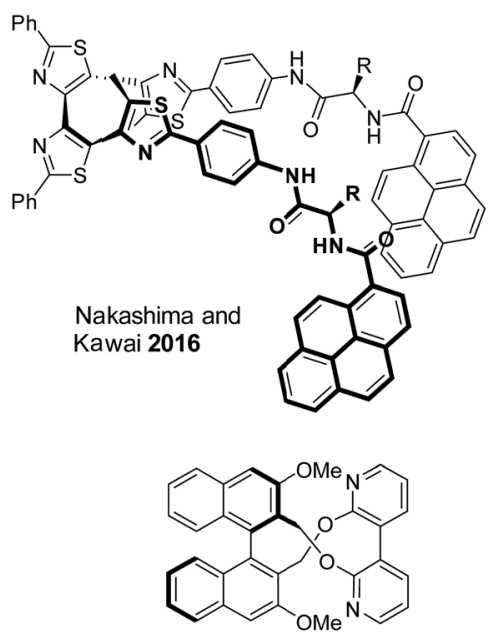

Takaishi and Ema 2018
Fig. 1 Selected examples of purely organic reversible CPL switches. 
helical tetrathiazole upon photoisomerization. ${ }^{20}$ Ito, Imai, Asami and coworkers synthesized a concentration dependent pyrene-based CPL switch. ${ }^{18}$ Finally, Takaishi and Ema developed a macrocyclic acid/base triggered on/off CPL switch comprising of a binaphthyl linked to a 3,3'-bipyridyl moiety. ${ }^{21}$

In this framework, our group recently reported the efficient large-scale synthesis of macrocycles using simple cyclic ethers and diazoketoester 1 as reagents. ${ }^{22}$ Resulting unsaturated derivatives, denominated 18C6, 18C4 or 16C4 (Scheme 1), react with aromatic amines to form chiral functionalized scaffolds 2 through tandem amidation/olefin transposition processes (Fig. 2, top). ${ }^{23}$ Of importance for the current study, products 2 assume a limited number of restricted conformations with the carbonyl groups facing outwards and the two aromatic units at immediate spatial proximity to each other (Fig. 2).$^{24}$

If pyrenes are introduced as aromatic nuclei, then strong telltale excimer fluorescence (EF) is observed. ${ }^{25}$ However, upon binding of monovalent or divalent cations, major conformational

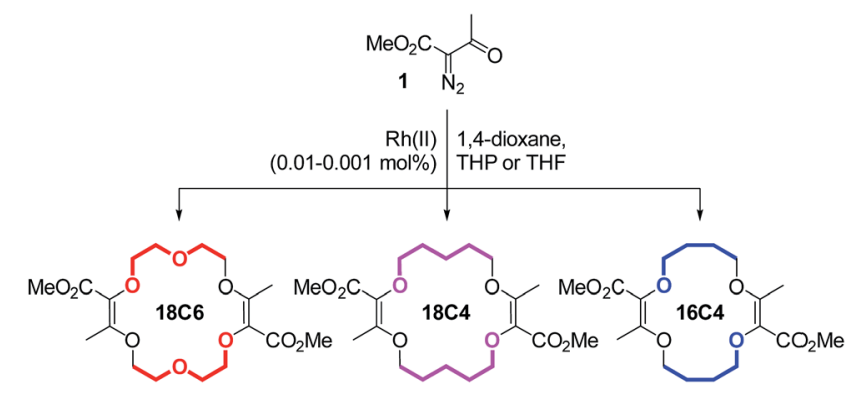

Scheme 1 Synthesis of unsaturated macrocycles 18C6,18C4 and $16 \mathrm{C} 4$.
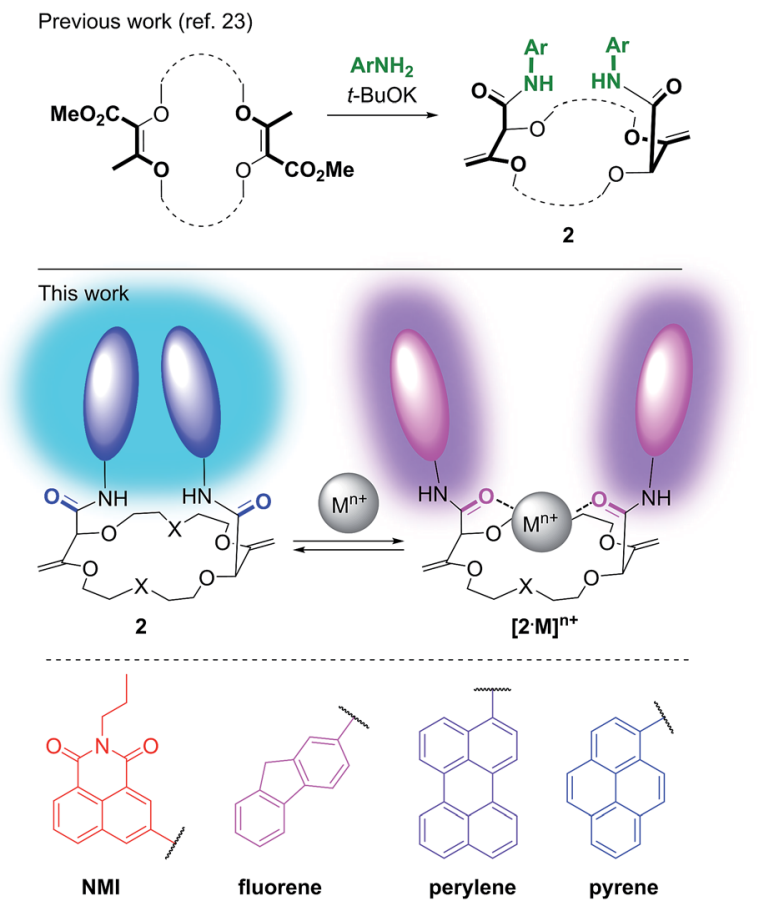

Fig. 2 Synthesis of chiral macrocycles (top). Proposed conformational change upon cation binding (middle). Selected fluorophores (bottom). changes occur. Presumably, the amide bonds rotate and the resulting inward orientation of the $\mathrm{C}=\mathrm{O}$ bonds helps complex the cations. ${ }^{26}$ As a consequence, the aromatic rings part from each other, elongating the distance between the chromophores (Fig. 2). The excimer fluorescence is quenched and only the characteristic emission of (monomeric) pyrene is observed.

Herein, we took advantage of this unique ability to induce and cancel excimer fluorescence to develop a family of enantiopure chiroptical switches with a large and tunable wavelength emission range. For this purpose, a variety of fluorophores - namely NMI (NMI: $N$-propyl-1,8-naphthalene monoimide), fluorene, perylene and pyrene (Fig. 2, bottom) were selected and introduced on different macrocyclic scaffolds (10 examples). After resolution by chiral stationary phase (CSP) HPLC, high CPL response ( $g_{\text {lum }}$ up to $1.7 \times 10^{-2}$ ) allied with intense excimer fluorescence was demonstrated. In presence of metal ions $\left(\mathrm{Na}^{+}, \mathrm{Ba}^{2+}\right)$, the ECD, EF and CPL responses are strongly affected. While the ECD signals can be almost completely reversibly inverted upon the complexation/ decomplexation of metal ions in a typical binary +/response, the CPL signal is reversibly quenched establishing a rare combined reversible switching of ECD and extinction of CPL behavior for the designed macrocycles.

\section{Results and discussion}

\section{Fluorescent macrocycles: synthesis and resolution}

Unsaturated macrocycles 18C6, 18C4 and 16C4 (Scheme 1) were selected as building blocks. The synthesis of 18C6 was achieved on multigram scale by Rh(II)-catalyzed (0.001 mol\%) decomposition of $\alpha$-diazo- $\beta$-keto ester 1 in 1,4-dioxane as solvent and reactant (Scheme $\mathrm{S} 1 \dagger$ ). ${ }^{22 a}$ Application of this procedure to the gram scale preparation of 18C4 and 16C4 was possible with a larger amount of catalyst $(0.01 \mathrm{~mol} \%)$ and THP/THF as cyclic ethers respectively (Scheme S1†). ${ }^{27}$ Treatment of the three derivatives with aromatic amines (3.0 equiv. $)^{28}$ under strongly basic conditions ( $t$-BuOK, 4.0 equiv.) afforded the corresponding functionalized macrocycles in moderate to good yields (17$80 \%$, Fig. 2 and 3). ${ }^{23,25 b}$ In this manner, 1-amino-pyrene, 3amino-NMI, 2-amino-fluorene ${ }^{29}$ and 1-amino-perylene were introduced effectively. In all cases, the diastereoselectivity of the reaction was excellent (d.r. $>49: 1$ ) in favor of the chiral (racemic) diastereomers.

With the ten compounds in hand, the CSP-HPLC resolution was performed. A general and practical protocol was developed using eluents in which the macrocyclic derivatives are (highly) soluble to render the semi-preparative enantiomer separation operational. It was found that CHIRALPAK® IG as CSP and a mixture of $\mathrm{CH}_{2} \mathrm{Cl}_{2}\left(+0.1 \% \mathrm{Et}_{2} \mathrm{NH}\right)$ and $\mathrm{CH}_{3} \mathrm{CN}\left(+0.1 \% \mathrm{Et}_{2} \mathrm{NH}\right)$ as mobile phase was particularly efficient. ${ }^{30}$ Good selectivity factors $(\alpha)$ and pure samples of both enantiomers were always obtained (see Table S1†).

\section{UV-Vis absorption and switching of ECD properties}

With enantiopure materials in hand, the UV-Vis absorption and ECD spectra were recorded in $\mathrm{CH}_{3} \mathrm{CN}$ for 18C6-based 


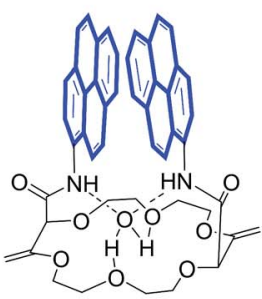

pyrene-18C6, 60\%

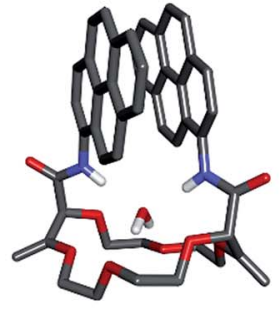

pyrene-18C6, X-ray

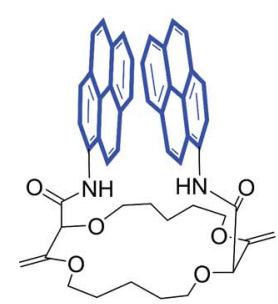

pyrene-18C4, $70 \%$

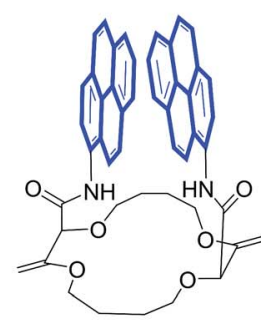

pyrene-16C4, $80 \%$

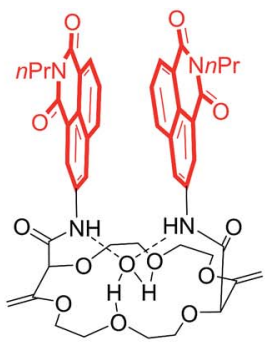

NMI-18C6, 17\%

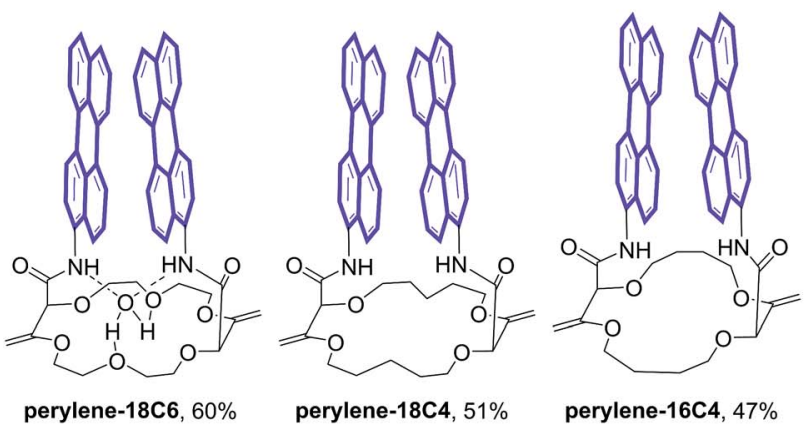

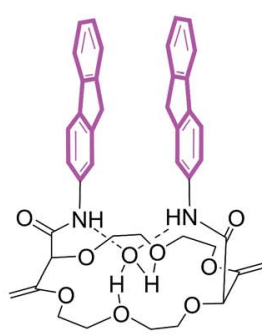

fluorene-18C6, $38 \%$

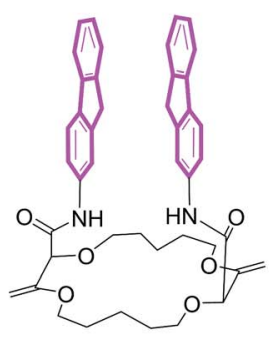

fluorene-18C4, $19 \%$

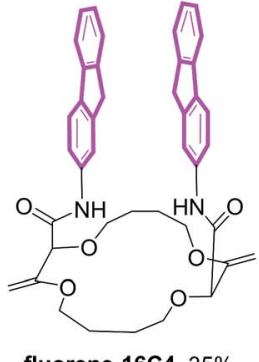

fluorene-16C4, 35\%

Fig. 3 Functionalized macrocycles of study.

macrocycles and in $\mathrm{CH}_{2} \mathrm{Cl}_{2}$ for 18C4- and 16C4-based derivatives (Fig. 4 and S5-S17†); the reason for the solvent swap will become clear later in the manuscript. The perylene and fluorene series (Fig. 4B and C, S16B and C and S17B and C $\dagger$ ) displayed strong exciton couplets (bisignate ECD features) in correspondence with their most red-shifted absorption band, which is broad and intense in both cases. The exciton couplets are symmetric (the two component bands have similar integrals) and show clear vibrational sub-structures. Interestingly and somewhat surprisingly, the ECD spectra of pyrene derivatives displayed only a monosignate signal for the first absorption band (Fig. 4A, S16A and S17A $\dagger$ ). In fact, an exciton coupling was expected for this transition in view of the crystallographic geometry that indicates a spatial proximity and a skewed arrangement of the two aromatic moieties..$^{31}$ For these derivatives, some discrepancy between solution and solid state structures is likely to occur, including the possibility of a dynamic reorientation of the aromatic rings. Finally, NMI-18C6 displayed intense exciton couplets in the region between 230 and $300 \mathrm{~nm}$ (Fig. 4D), where strong absorptions occur, and very weak ECD bands on the right edge of the spectrum, in correspondence with weaker absorption bands.

ECD spectra of 18C6 macrocycles were simulated by means of time-dependent density functional theory (TDDFT) calculations $^{32}$ with the aim of interpreting the observed chiroptical properties and possibly establishing the absolute configuration of the macrocycles with respect to the elution order. Input geometries were generated starting from the available X-ray structure of pyrene-18C6 with $(S, S)$ configuration (bound to one water molecule), and optimized with DFT using the M06-2X functional with D3 dispersion correction, ${ }^{33}$ to properly describe intermolecular $\pi$-stacking. TDDFT calculations were run with different combinations of functionals, basis sets and environment description (see ESI $\dagger$ ). From the start, the attempted accurate simulation of the ECD spectra of the functionalized 18C6-based macrocycles was a formidable task because of (a) the molecular size, associated with the complexity of the involved chromophores, and (b) the conformational ambiguity. In fact, even assuming a strong preference for $\pi$-stacked structures in solution, the two $N$-aryl amide moieties can assume several reciprocal arrangements, by varying the orientation of the macrocycle- $\mathrm{C}(=\mathrm{O})$ and $N-$ aryl bonds, which are likely to produce very different ECD spectra. This is illustrated in Fig. S36† for fluorene-18C6. The most immediate result was that, for any given input structure, a strong exciton-coupled ECD spectrum was calculated in each case. This is easily appreciable in the DFT-optimized geometries for fluorene-18C6 in which the aromatic rings are partially stacked over each other defining a negative or positive exciton chirality, depending on the reciprocal arrangement of the fluorene rings, associated with strong negative and positive ECD couplet, respectively (Fig. S36†).

If the relative energies from DFT optimizations are entirely trusted, the Boltzmann-weighted calculated ECD spectrum for $(S, S)$-fluorene-18C6 consists into a negative exciton couplet at long wavelength, as found experimentally for the $1^{\text {st }}$ eluted enantiomer (Fig. 4C). When a clear-cut couplet is not apparent from experimental spectra, like for the red-shifted bands of pyrene-18C6 or NMI-18C6 (Fig. 4A and D), the reason must be sought in the coexistence of multiple conformations with different arrangements of the aromatic rings which yield ECD spectra canceling each other in the red-shifted portion of the spectrum, as illustrated in Fig. S37. $\uparrow$ The calculated ECD profiles suggest that for pyrene-, perylene- and NMI-18C6, the $2^{\text {nd }}$ eluted enantiomers have $(S, S)$ configuration. 

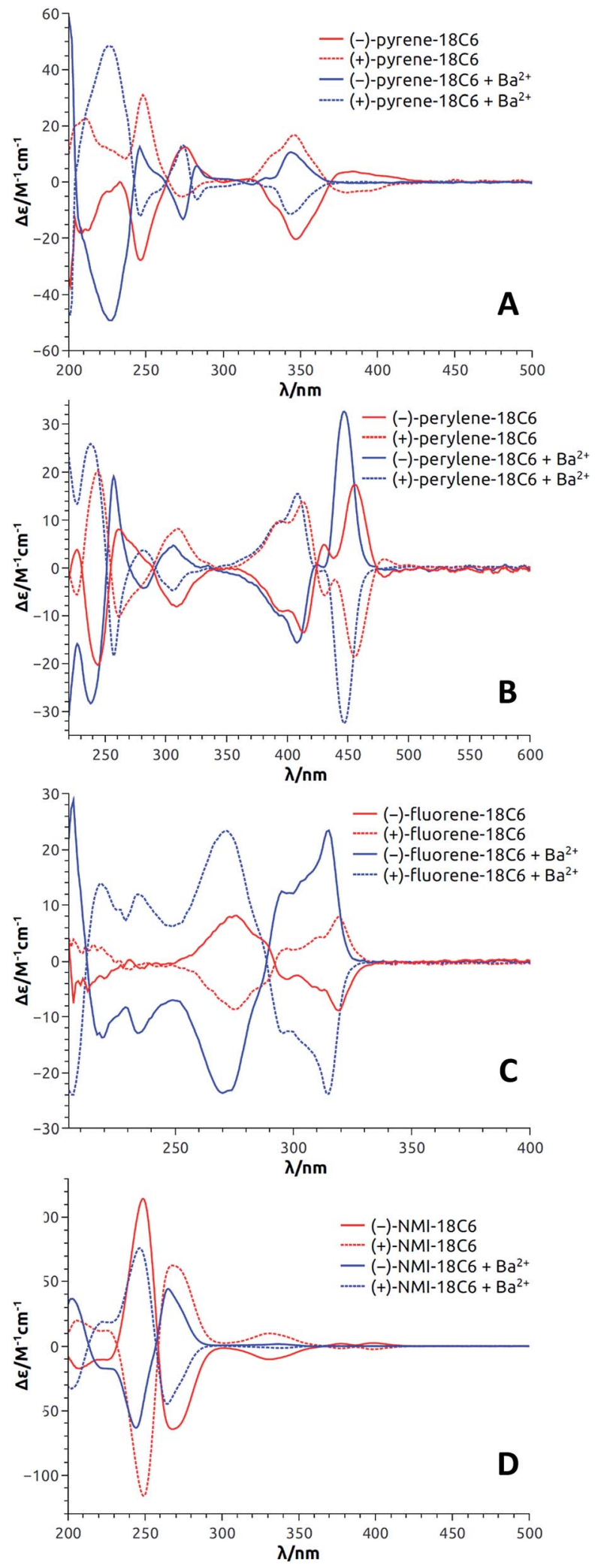

Fig. 4 ECD spectra $\left(\mathrm{CH}_{3} \mathrm{CN}\right)$ of both enantiomers of pyrene-18C6 (A), perylene-18C6 (B), fluorene-18C6 (C) and NMI-18C6 (D) without (red) and with (blue) $\mathrm{Ba}\left(\mathrm{ClO}_{4}\right)_{2}$.
In the presence of monovalent or divalent cations, very different spectroscopic results were observed. Experimentally, it was necessary to use two different sets of conditions depending on the nature of the macrocyclic cores. Within the $18 \mathrm{C6}$ series, studies were conducted in acetonitrile with $\mathrm{Ba}\left(\mathrm{ClO}_{4}\right)_{2}$ as metal ion source. ${ }^{25 a}$ With 18C4- and 16C4-based macrocycles, conditions had to be changed to (soluble) $\mathrm{NaBAr}_{\mathrm{F}}$ in $\mathrm{CH}_{2} \mathrm{Cl}_{2}$ to achieve a modulation of the optical properties. ${ }^{34}$ While little effect was observed in UV-Vis absorption, strong variations of the signals were obtained in ECD in the presence of $\mathrm{Na}^{+}$or $\mathrm{Ba}^{2+}$ ions; the chiroptical spectroscopy being particularly sensitive to conformational rearrangements. ${ }^{35}$ All changes were quantified using eqn (1), in which $\Delta \varepsilon_{\text {(cation) }}$ and $\Delta \varepsilon_{\text {(without) }}$ represent the normalized ECD intensity (in $\Delta \varepsilon$ ) of the compound in presence or absence of tested metal ions, respectively. The $\delta \Delta \varepsilon$ values are summarized in Table 1.

$$
\delta \Delta \varepsilon=\left|\Delta \varepsilon_{\text {(cation) }}-\Delta \varepsilon_{\text {(without) }}\right|
$$

The results with pyrene-18C6, perylene-18C6, fluorene-18C6 and NMI-18C6 are first discussed; the spectra for each enantiomeric series being presented in Fig. 4. Importantly and interestingly, for each compound, transition(s) can always be found for which the sign of the Cotton effect switches from positive to negative or vice versa. In the case of pyrene-18C6, the spectra undergo remarkable ECD sign reversal for almost all the observed transitions in the $200-500 \mathrm{~nm}$ range (Fig. 4A). With perylene-18C6, the ECD switch is less dramatic but still significant at $446 \mathrm{~nm}$ (Fig. 4B). It might be due to the bulkier nature of the perylene moiety, which does not allow for the necessary conformational freedom to attain a complete rearrangement upon addition of the barium salt. However, the fluorene-18C6 derivative presents not only the sign reversal in the exciton couplet region but also a strong increase of the intensity (Fig. 4C). For NMI-18C6, sign inversion is observed at a higher energy (around $260 \mathrm{~nm}$ ) for the exciton coupling-like feature (Fig. 4D). For completeness and as control experiments, the ECD behavior was also monitored in the presence of $\mathrm{NaBAr}_{\mathrm{F}}$ in $\mathrm{CH}_{2} \mathrm{Cl}_{2}$; variations upon addition of $\mathrm{Na}^{+}$were very similar to that with $\mathrm{Ba}^{2+}$ in this $\mathbf{1 8 C 6}$ series (Fig. S15 $\dagger$ ). With compounds pyrene-18C4, perylene-18C4 and fluorene-18C4, similar experiments were conducted but only with $\operatorname{NaBAr}_{F}$ (Fig. S16 $\dagger$ ). This was also the case for pyrene-16C4, perylene-16C4 and fluorene16C4 (Fig. S17 $\dagger$ ). Globally, the trends detailed above are reproduced for the macrocycles with all-carbon links between the polar units. A small difference is displayed by fluorene-16C4 which shows a couplet of different sign with respect to compounds fluorene-18C6 and fluorene-18C4. The intensity of this couplet increases but it does not change sign upon $\mathrm{Na}^{+}$ addition.

\section{Switching of fluorescence and CPL properties}

Satisfactorily, all functionalized macrocycles displayed excimer fluorescence (Fig. 5 and S5-S14 $\dagger$ ). As expected, the emissions are red shifted compared to "monomer" emissions; a series of simple neopentylcarboxamides being used as references (see 
Table 1 Chiroptical properties $\left(g_{\mathrm{abs}}, \delta \Delta \varepsilon\right.$ and $\left.g_{\text {lum }}\right)$, without and with cations ${ }^{a, b}$

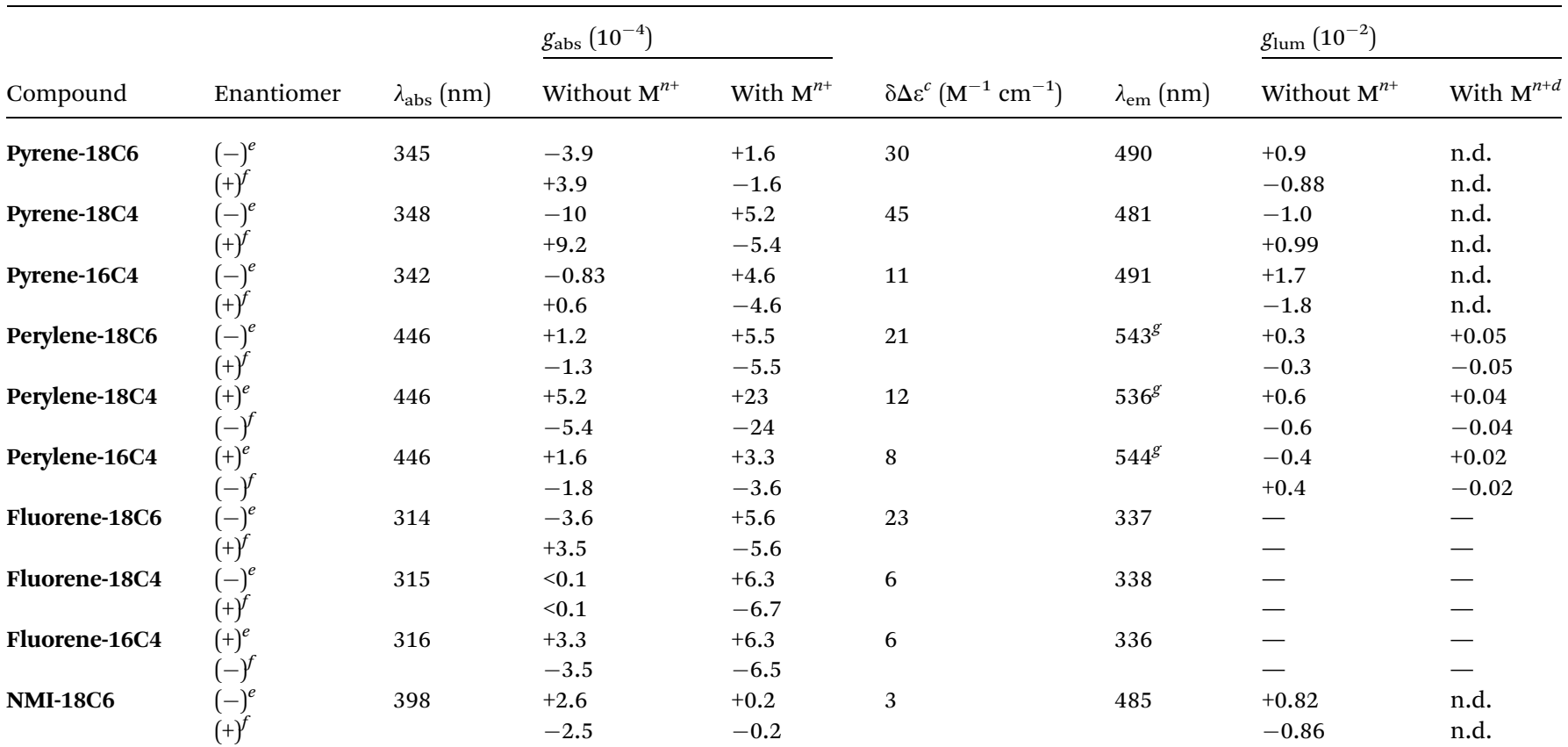

${ }^{a} \mathrm{Ba}\left(\mathrm{ClO}_{4}\right)_{2} / \mathrm{CH}_{3} \mathrm{CN}$ system used for $18 \mathrm{C} 6$ derivatives, $\mathrm{NaBAr}_{\mathrm{F}} / \mathrm{CH}_{2} \mathrm{Cl}_{2}$ system used for $18 \mathrm{C} 4$ and $16 \mathrm{C} 4$ compounds, $g_{\text {abs }}$ and $g_{\text {lum }}$ are calculated on reported $\lambda_{\text {abs }}$ and $\lambda_{\text {em }}$ respectively (unless otherwise stated). ${ }^{b}$ n.d. $=$ not detected. ${ }^{c}$ As defined by eqn (1). ${ }^{d}$ Determined on the monomer emission band. ${ }^{e}$ First eluted. ${ }^{f}$ Second eluted. ${ }^{g} \lambda$ associated with excimer emission.

the ESI $\uparrow$ for the synthesis and characterizations). In the pyrene series, the excimer band is particularly intense at $490 \mathrm{~nm}\left(\lambda_{\max }\right)$ and, for the $\mathbf{1 8 C 4}$ moiety specifically, the interaction is so strong that the emission band of the monomer is no longer observable. With compounds carrying perylene moieties, a weaker and broad EF is observed around $540 \mathrm{~nm}$. Fluorene derivatives present very strong EF (337 nm) in comparison to the almost non-detectable monomer band. Finally, NMI-18C6 presents similar characteristics to perylene-18C6 although with a more intense excimer band $(485 \mathrm{~nm})$ in respect to the monomer. Globally, with this family of compounds, the excimer fluorescence spans a spectral window from UV to green-yellow (ca. $300 \mathrm{~nm}$ to $650 \mathrm{~nm}$ ). Also, while $18 \mathrm{C6}$ and $\mathbf{1 8 C 4}$ derivatives display strong EF contribution with respect to the monomer fluorescence, the effect is less pronounced with $16 \mathrm{C4}$ analogues. Actually, for the smaller ring size compounds, solid-state analysis indicates a non-parallel arrangement of the aromatic moieties (Fig. 6) and hence the possible reduction in EF efficiency.

Upon addition of either $\mathrm{Na}^{+}$or $\mathrm{Ba}^{2+}$ cations, full quenching of the excimer fluorescence is observed, and this with all fluorophores, independently of their macrocycle size and nature. Only the sharper monomer emissions remain. This observation is in agreement with the proposed conformational rearrangement upon cation complexation (vide supra, Fig. 2). Emission maxima $\left(\lambda_{\max }\right)$ and fluorescence quantum yields $(\phi)$ of all compounds are reported in Table S3. $\dagger$

With these results in hand demonstrating (very) effective EF, circularly polarized luminescence studies were started expecting, in accordance with previously reported examples, ${ }^{\mathbf{1 8 , 2 0 , 3 6 , 3 7}}$ efficient CPL emission allied with the excimer band. In fact and as expected, pyrene, perylene and NMI derivatives presented strong excimer-associated CPL (Fig. 7, S18 and S19†); a weak contribution from the monomer circularly polarized emission being observed only in the perylene series (Fig. 7B, S18B and $\mathrm{S} 19 \mathrm{~B} \dagger)$. In the fluorene series, due to the technical limitations of the CPL apparatus in the UV region, CPL spectra could not be measured. To quantify the circular polarization degree of the emission, the luminescence dissymmetry factor $g_{\text {lum }}$ was used as defined by eqn (2)

$$
g_{\text {lum }}=2 \frac{I_{\mathrm{L}}-I_{\mathrm{R}}}{I_{\mathrm{L}}+I_{\mathrm{R}}}
$$

where $I_{\mathrm{L}}$ and $I_{\mathrm{R}}$ correspond to left and right circularly polarized component of the emission respectively. The $g_{\text {lum }}$ factors for all tested compounds are reported in Table 1.

For most compounds, the luminescence dissymmetry factors calculated on the CPL/fluorescence maxima are around $10^{-2}$. These $g_{\text {lum }}$ values are in the upper range recorded for single (i.e. non-aggregated) organic molecules, ${ }^{38}$ and in line with that of excimer bands formed by pyrenes, ${ }^{18,20,39}$ naphthalimides ${ }^{40}$ and naphthalene diimides ${ }^{41}$ or perylene bisimides. ${ }^{42}$ In the perylene series, $g_{\text {lum }}$ values not higher than $10^{-3}$ were obtained. The decrease of the observed dissymmetry factor is possibly due to the contribution of the weakly polarized perylene monomer emission (Table 1). ${ }^{43}$

Since, in these systems, CPL stems from the excimer, a photophysical state formed only in the excited state, the $g_{\text {lum }}$ factor does not need to be related to the ECD dissymmetry factor $\left(g_{\text {abs }}\right.$ $=\Delta \varepsilon / \varepsilon$ ) measured on the first Cotton effect, as it would be expected if the same electronic states were involved in absorption 

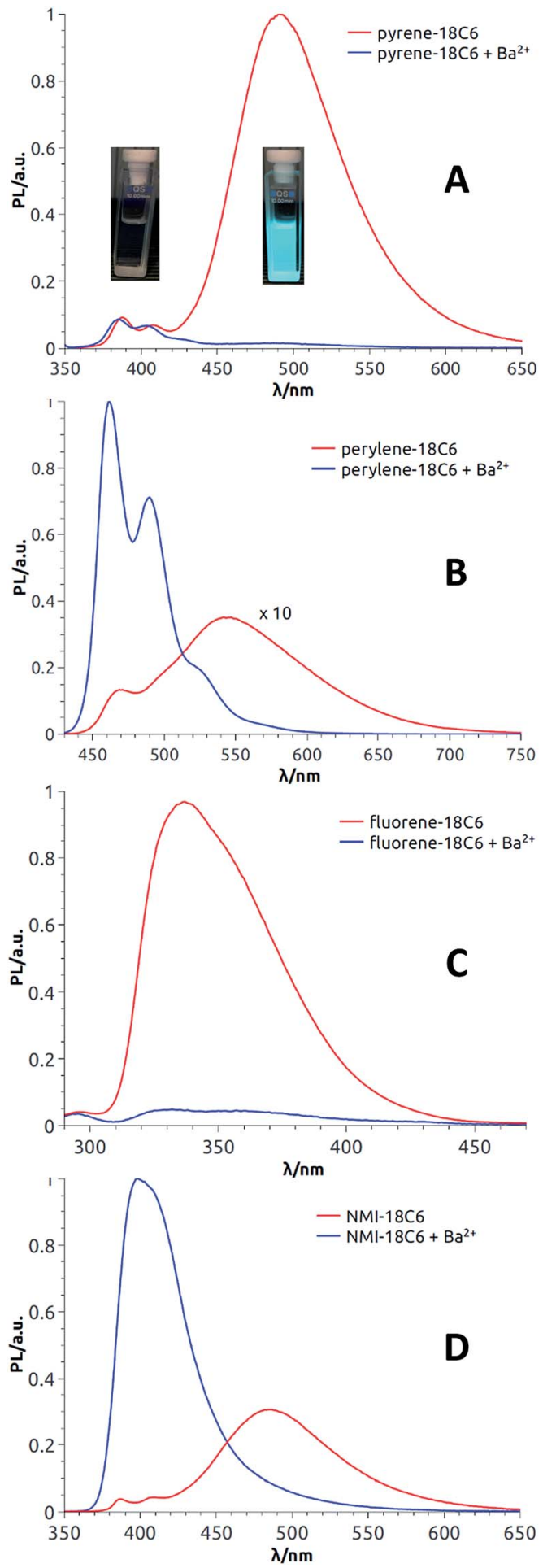

Fig. 5 Fluorescence spectra $\left(\mathrm{CH}_{3} \mathrm{CN}\right)$ of pyrene-18C6 (A), perylene$18 \mathrm{C} 6(\mathrm{~B})$, fluorene-18C6 (C) and NMI-18C6 (D) without (red) and with (blue) $\mathrm{Ba}\left(\mathrm{ClO}_{4}\right)_{2}$. In panel A: picture of pyrene-18C6 without (left) and with (right) $\mathrm{Ba}^{2+}$ under UV irradiation $(366 \mathrm{~nm}$ ).

and emission. ${ }^{44}$ Indeed, in the present case, $g_{\text {abs }}$ values are in the $10^{-3}$ to $10^{-4}$ range while $g_{\text {lum }}$ factors, calculated on the CPL/ fluorescence maxima, are higher by at least one order of magnitude $\left(10^{-2}\right)$.

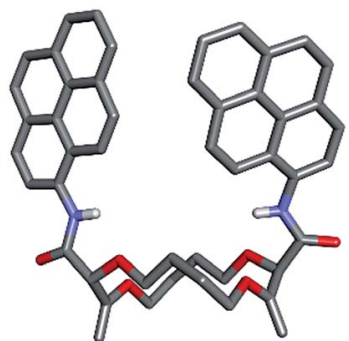

Fig. 6 Stick view of the crystal structure of pyrene-16C4.
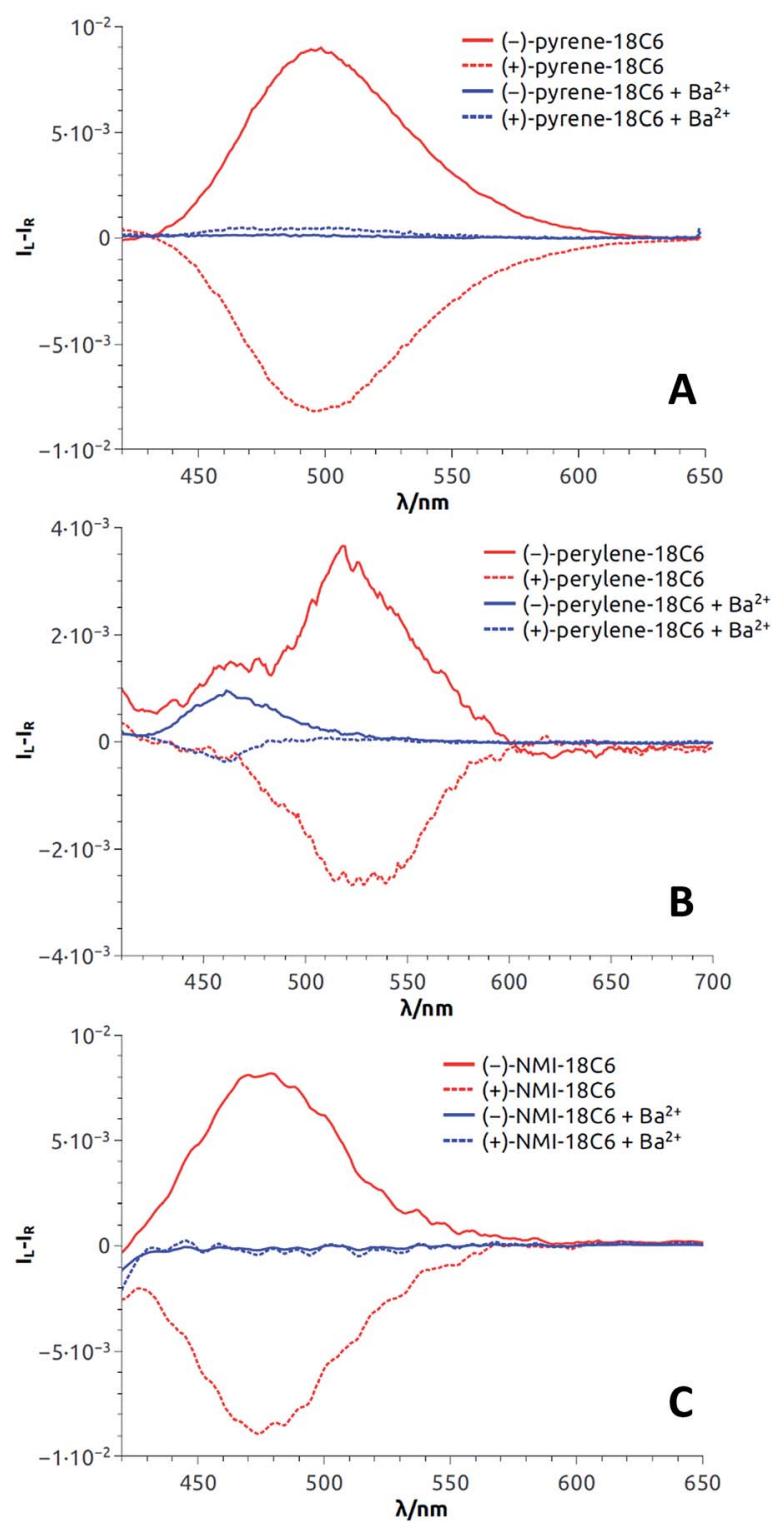

Fig. $7 \mathrm{CPL}$ spectra $\left(\mathrm{CH}_{3} \mathrm{CN}\right)$ of both enantiomers of pyrene-18C6 (A), perylene-18C6 (B) and NMI-18C6 (C) without (red) and with (blue) $\mathrm{Ba}\left(\mathrm{ClO}_{4}\right)_{2}$.

Then, as it could be expected from the EF experiments, full quenching of CPL signals was achieved for pyrene and NMI derivatives upon addition of either $\mathrm{Na}^{+}$or $\mathrm{Ba}^{2+}$ cations (Fig. 7A and C, S18A and S19A $\dagger$ ). In these cases, the monomer bands are 


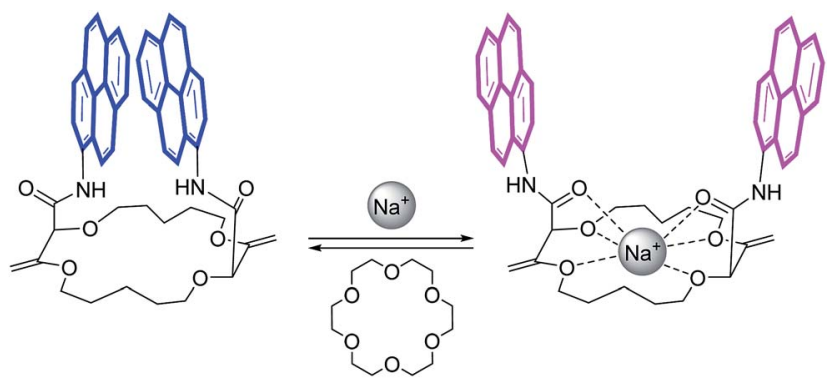

Scheme 2 Model for reversible switching.

too weak to be detected. However, with perylene compounds (Fig. 7B, S18B and S19B $\dagger$ ), it was possible to measure a weak CPL signal allied to the strong monomer emission ( $g_{\text {lum }}$ up to 5 $\times 10^{-4}$ ), in the expected $g_{\text {lum }}$ range for (weakly) chirallyperturbed fluorophores.

\section{ECD/CPL reversibility}

All these experiments bode well for the development of effective +/- ECD and on/off CPL switches upon the establishment of reversible metal complexation conditions with the compounds at hand. In view of the strong binding of barium cations to $18 \mathrm{C} 6$ derivatives in acetonitrile, care was taken to study the reversibility of the complexation of monovalent cations instead. ${ }^{25 a}$ For practical reasons, we selected the conditions already established, i.e. $\mathrm{NaBAr}_{\mathrm{F}}$ in $\mathrm{CH}_{2} \mathrm{Cl}_{2}$ and decided to use regular 18-Crown-6 as a cation scavenger (Scheme 2). ${ }^{45}$

Equimolar amounts of $\mathrm{NaBAr}_{\mathrm{F}}$ and 18-Crown-6 solutions were thus successively added to dichloromethane solutions of the pyrene, perylene, fluorene and NMI fluorophores. After each addition, ECD, then fluorescence and finally CPL spectra were recorded. Data for the pyrene-18C4 derivative, selected as a representative example, are displayed in Fig. 8. Remaining data are reported in ESI (Fig. S20-S35). $\dagger$ As desired, upon addition of commercial 18-Crown-6 to the $\mathrm{Na}^{+}$adduct, it was possible to completely switch back the system and recover the ECD signals of the uncomplexed material (Fig. 8A). The switch experiment could be repeated over several cycles without significant signal loss or modification (e.g., Fig. $8 \mathrm{~B}$ at $348 \mathrm{~nm}$ ). Using the same $18 \mathrm{C} 4$ model, almost complete reversible switching - from excimer to monomer - was monitored by fluorescence (Fig. S30†). In CPL, these conditions led also to a very good recovery of the signal, over several cycles (Fig. 8C and D). Pyrene-18C4 may therefore be considered as a completely reversible +/- ECD and on/off CPL switch.

Appreciatively, using the same reagent combination, all pyrene and fluorene macrocycles behave as fully reversible ECD switches over several cycles (Fig. S20-S21 and S25-S27†). In CPL, pyrene-18C6 demonstrated reversibility in spite of an attenuation of the excimer luminescence intensity over the cycles (Fig. S34 $\dagger$ ). This trend is even more acute with analogous pyrene-16C4 that showed reversibility over only two cycles - presumably due to the strong photobleaching occurring under the CPL measurement conditions for this compound (Fig. S35†). Finally, for perylene and NMI-18C6 derivatives, signal recovery to the expected intensity and shape could not be achieved over successive additions of $\mathrm{NaBAr}_{\mathrm{F}}$ and 18-Crown-6 (Fig. S22-S24 and S28 $\dagger$ ). The reversibility of the CPL signal was hence not studied for these cases.
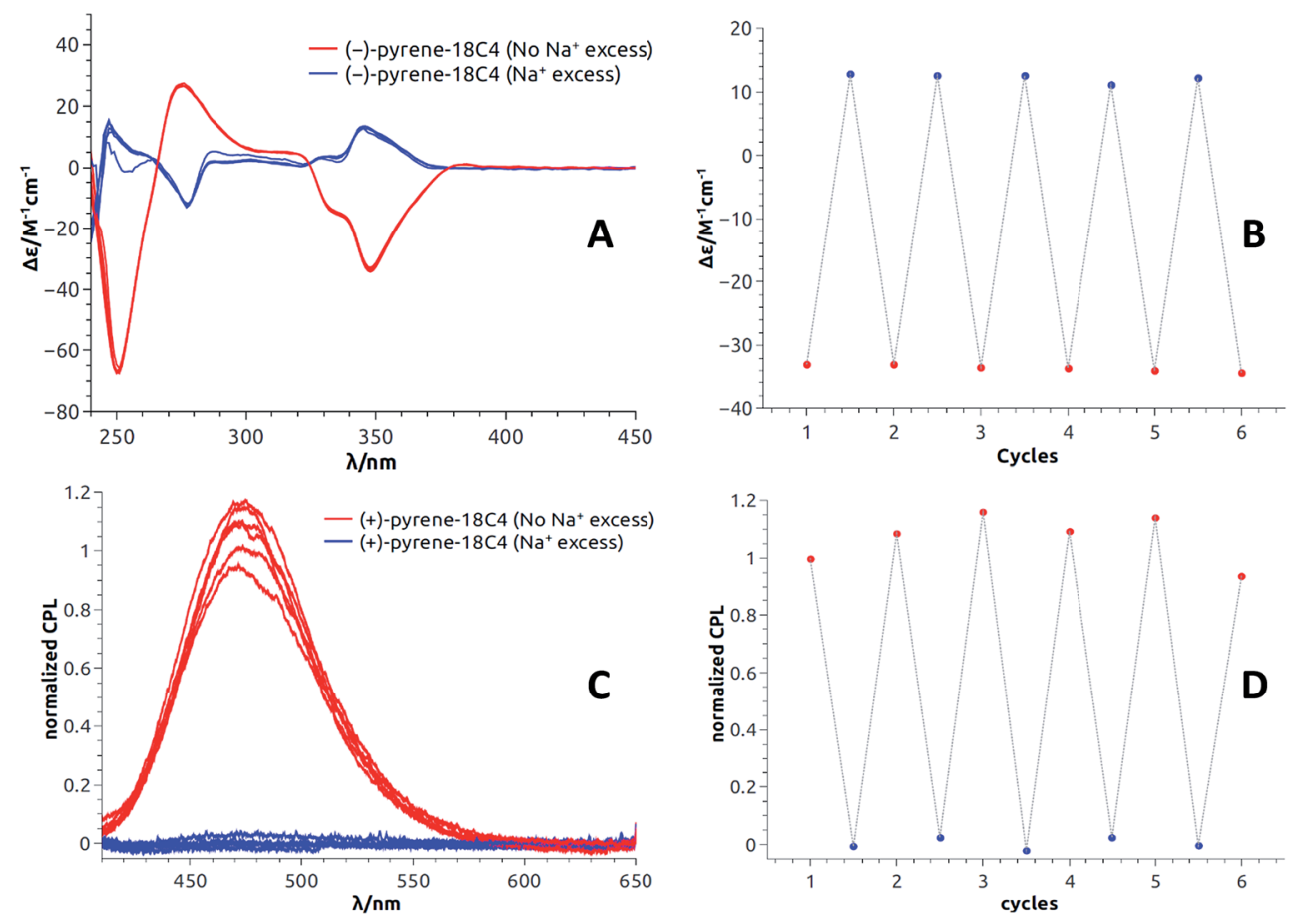

Fig. 8 Pyrene-18C4 $\left(\mathrm{CH}_{2} \mathrm{Cl}_{2}\right)$ : Reversible ECD ((A) first eluted (-)-enantiomer) and intensities at $348 \mathrm{~nm}(\mathrm{~B})$. Reversible $\mathrm{CPL}$ ((C) second eluted (+)-enantiomer) and normalized intensities at $480 \mathrm{~nm}$ (D). 


\section{Conclusions}

In summary, we presented a series of readily prepared functionalized macrocycles showing efficient excimer luminescence - in different spectral regions - due to the spatial proximity of fluorophores held in place by the constrained macrocyclic structures. Using enantiopure materials, obtained through CSPHPLC resolution, a sign inversion of ECD signal(s) was demonstrated in all cases upon addition of cations like $\mathrm{Na}^{+}$or $\mathrm{Ba}^{2+}$. In addition, these molecules display a highly circularly polarized luminescence associated with the excimer fluorescence with $g_{\text {lum }}$ values up to $10^{-2}$, which can be quenched upon cation binding. For several systems, a complete recovery of ECD/CPL signals over several cycles was observed after reversible complexation of the cation, $\mathrm{Na}^{+}$specifically together with commercial 18-Crown-6 as sequester, giving rise to rare examples of allied +/- ECD and on/off CPL switches.

\section{Conflicts of interest}

There are no conflicts to declare.

\section{Abbreviation}

$\begin{array}{ll}\text { CPL } & \text { Circularly polarized luminescence } \\ \text { ECD } & \text { Electronic circular dichroism } \\ \text { EF } & \text { Excimer fluorescence } \\ \text { NMI } & N \text {-Propyl-1,8-naphthalene monoimide }\end{array}$

\section{Acknowledgements}

A. H., E. B., F. Z., S. P., C. B. and J. L. thank the University of Geneva and the Swiss NSF for financial support. M. G. thanks the Polish Ministry of Science and Higher Education ('Mobilnosc Plus' grant no. 1286/MOB/IV/2015/0) for the support. We thank Dr Pilar Franco and Mrs Assunta Green (Chiral Technologies, Illkirch, France) for their help and advices. We also acknowledge the contribution of Mr Mahesh Vishe for the obtaining of the monocrystals of pyrene-16C4. We thank the Sciences Mass Spectrometry (SMS) platform at the Faculty of Sciences, University of Geneva. G. P. acknowledges the CINECA award under the ISCRA initiative, for the availability of high performance computing resources and support.

\section{Notes and references}

1 J. R. Brandt, F. Salerno and M. J. Fuchter, Nat. Rev. Chem., 2017, 1, 0045.

2 C. Wang, H. Fei, Y. Qiu, Y. Yang, Z. Wei, Y. Tian, Y. Chen and Y. Zhao, Appl. Phys. Lett., 1999, 74, 19-21.

3 B. L. Feringa, Acc. Chem. Res., 2001, 34, 504-513.

4 (a) D. M. Lee, J. W. Song, Y. J. Lee, C. J. Yu and J. H. Kim, Adv. Mater., 2017, 29, 1700907; (b) F. Zinna, M. Pasini, F. Galeotti, C. Botta, L. Di Bari and U. Giovanella, Adv. Funct. Mater., 2017, 27, 1603719; (c) J. R. Brandt, X. Wang, Y. Yang,
A. J. Campbell and M. J. Fuchter, J. Am. Chem. Soc., 2016, 138, 9743-9746; (d) F. Zinna, U. Giovanella and L. Di Bari, Adv. Mater., 2015, 27, 1791-1795; (e) Y. Yang, R. Correa da Costa, D. M. Smilgies, A. J. Campbell and M. J. Fuchter, Adv. Mater., 2013, 25, 2624-2628.

5 Y. Yang, R. Correa da Costa, M. J. Fuchter and A. J. Campbell, Nat. Photonics, 2013, 7, 634-638.

6 (a) V. Kiran, S. P. Mathew, S. R. Cohen, I. Hernández Delgado, J. Lacour and R. Naaman, Adv. Mater., 2016, 28, 1957-1962; (b) P. C. Mondal, N. Kantor-Uriel, S. P. Mathew, F. Tassinari, C. Fontanesi and R. Naaman, Adv. Mater., 2015, 27, 1924-1927; (c) B. Göhler, V. Hamelbeck, T. Markus, M. Kettner, G. Hanne, Z. Vager, R. Naaman and H. Zacharias, Science, 2011, 331, 894-897.

7 (a) H. Isla and J. Crassous, C. R. Chim., 2016, 19, 39-49; (b) Z. Dai, J. Lee and W. Zhang, Molecules, 2012, 17, 1247-1277. 8 (a) N. Saleh, B. Moore II, Srebro, N. Vanthuyne, L. Toupet, J. A. G. Williams, C. Roussel, K. K. Deol, G. Muller, J. Autschbach and J. Crassous, Chem.-Eur. J., 2015, 21, 1673-1681; (b) M. Srebro, E. Anger, B. Moore II, N. Vanthuyne, C. Roussel, R. Réau, J. Autschbach and J. Crassous, Chem.-Eur. J., 2015, 21, 17100-17115; (c) E. Anger, M. Srebro, N. Vanthuyne, C. Roussel, L. Toupet, J. Autschbach, R. Réau and J. Crassous, Chem. Commun., 2014, 50, 2854-2856; (d) E. Anger, M. Srebro, N. Vanthuyne, L. Toupet, S. Rigaut, C. Roussel, J. Autschbach, J. Crassous and R. Réau, J. Am. Chem. Soc., 2012, 134, 15628-15631.

9 (a) W. Miao, S. Wang and M. Liu, Adv. Funct. Mater., 2017, 27, 1701368; (b) W. Miao, L. Qin, D. Yang, X. Jin and M. Liu, Chem.-Eur. J., 2015, 21, 1064-1072; (c) W. Zhao, D. Wang, H. Lu, Y. Wang, X. Sun, S. Dong and J. Hao, Langmuir, 2015, 31, 2288-2296; (d) W. Miao, L. Zhang, X. Wang, H. Cao, Q. Jin and M. Liu, Chem.-Eur. J., 2013, 19, 3029-3036.

10 M.-J. Kim, S.-J. Yoo and D.-Y. Kim, Adv. Funct. Mater., 2006, 16, 2089-2094.

11 (a) S. Shuvaev, M. A. Fox and D. Parker, Angew. Chem., Int. Ed., 2018, 57, 7488-7492; (b) F. Zinna and L. Di Bari, Chirality, 2015, 27, 1-13; (c) R. Carr, N. H. Evans and D. Parker, Chem. Soc. Rev., 2012, 41, 7673-7686; (d) G. Muller, Dalton Trans., 2009, 9692-9707.

12 (a) C. Liu, D. Yang, Q. Jin, L. Zhang and M. Liu, Adv. Mater., 2016, 28, 1644-1649; (b) Y. Qiu, P. Chen, P. Guo, Y. Li and M. Liu, Adv. Mater., 2008, 20, 2908-2913; (c) G. Bottari, D. A. Leigh and E. M. Pérez, J. Am. Chem. Soc., 2003, 125, 13360-13361.

13 (a) W. Liu, D. Cao, J. Peng, H. Zhang and H. Meier, Chem.Asian J., 2010, 5, 1896-1901; (b) F.-L. Hu, H.-F. Wang, D. Guo, H. Zhang, J.-P. Lang and J. E. Beves, Chem. Commun., 2016, 52, 7990-7993.

14 (a) J. R. Brandt, L. Pospíšil, L. Bednárová, R. C. da Costa, A. J. P. White, T. Mori, F. Teplý and M. J. Fuchter, Chem. Commun., 2017, 53, 9059-9062; (b) M. Cortijo, C. Viala, T. Reynaldo, L. Favereau, I. Fabing, M. Srebro-Hooper, J. Autschbach, N. Ratel-Ramond, J. Crassous and J. Bonvoisin, Inorg. Chem., 2017, 56, 4555-4567; (c) D. Li, Z. Y. Wang and D. Ma, Chem. Commun., 2009, 1529-1531; (d) J. W. Canary, Chem. Soc. Rev., 2009, 38, 747-756; (e) 
J. W. Canary and S. Zahn, Trends Biotechnol., 2001, 19, 251255.

15 (a) X.-P. Zhang, J.-F. Mei, J.-C. Lai, C.-H. Li and X.-Z. You, J. Mater. Chem. C, 2015, 3, 2350-2357; (b) M. Fujiki, J. Am. Chem. Soc., 2000, 122, 3336-3343.

16 S. Pascal, C. Besnard, F. Zinna, L. Di Bari, B. Le Guennic, D. Jacquemin and J. Lacour, Org. Biomol. Chem., 2016, 14, 4590-4594.

17 (a) M. Gon, Y. Morisaki and Y. Chujo, Chem. Commun., 2017, 53, 8304-8307; (b) S. P. Morcillo, D. Miguel, L. Á. de Cienfuegos, J. Justicia, S. Abbate, E. Castiglioni, C. Bour, M. Ribagorda, D. J. Cárdenas, J. M. Paredes, L. Crovetto, D. Choquesillo-Lazarte, A. J. Mota, C. M. Carmen, G. Longhi and J. M. Cuerva, Chem. Sci., 2016, 7, 56635670; (c) H. Maeda, Y. Bando, K. Shimomura, I. Yamada, M. Naito, K. Nobusawa, H. Tsumatori and T. Kawai, J. Am. Chem. Soc., 2011, 133, 9266-9269.

18 S. Ito, K. Ikeda, S. Nakanishi, Y. Imai and M. Asami, Chem. Commun., 2017, 53, 6323-6326.

19 Cuerva, Miguel and coworkers reported recently a ratiomeric probe based on CPL using a bispyrene derived structure: P. Reiné, J. Justicia, S. P. Morcillo, S. Abbate, B. Vaz, M. Ribagorda, Á. Orte, L. Álvarez de Cienfuegos, G. Longhi, A. G. Campaña, D. Miguel and J. M. Cuerva, J. Org. Chem., 2018, 83, 4455-4463.

20 Y. Hashimoto, T. Nakashima, D. Shimizu and T. Kawai, Chem. Commun., 2016, 52, 5171-5174.

21 K. Takaishi, M. Yasui and T. Ema, J. Am. Chem. Soc., 2018, 140, 5334-5338.

22 (a) D. Poggiali, A. Homberg, T. Lathion, C. Piguet and J. Lacour, ACS Catal., 2016, 6, 4877-4881; (b) M. Vishe, R. Hrdina, L. Guénée, C. Besnard and J. Lacour, Adv. Synth. Catal., 2013, 355, 3161-3169; (c) W. Zeghida, C. Besnard and J. Lacour, Angew. Chem., Int. Ed., 2010, 49, 7253-7256.

23 M. Vishe, R. Hrdina, A. I. Poblador-Bahamonde, C. Besnard, L. Guénée, T. Bürgi and J. Lacour, Chem. Sci., 2015, 6, 49234928.

24 See ref. 23 for a more detailed discussion about the geometry of the crown ethers.

25 (a) M. Vishe, T. Lathion, S. Pascal, O. Yushchenko, A. Homberg, E. Brun, E. Vauthey, C. Piguet and J. Lacour, Helv. Chim. Acta, 2018, 101, e1700265; (b) Z. Jarolímová, M. Vishe, J. Lacour and E. Bakker, Chem. Sci., 2016, 7, 525533.

26 This rotation can be circumvented in cryptands derived from 2 in which the two aromatic moieties are linked through amide or urea bonds. See S. K. Ray, A. Homberg, M. Vishe, C. Besnard and J. Lacour, Chem.-Eur. J., 2018, 24, 2944-2951. 27 See ESI $\dagger$ for details on the synthesis.

28 In this study, the conditions of the tandem amidation/olefin transposition process were adapted to reduce the number of equivalents of aniline used and simplify purification procedures.

29 It has been noticed that the use of degassed THF for the synthesis of fluorene containing macrocycles synthesis increased substantially the yield due to the possible competitive oxidation of fluorene by $\mathrm{O}_{2}$ under basic conditions. See: X. Zhang, X. Ji, S. Jiang, L. Liu, B. L. Weeks and Z. Zhang, Green Chem., 2011, 13, 1891-1896.

30 We acknowledge the help of Chiral Technologies for the initial screening of columns and solvent conditions.

31 The X-ray structural analysis of pyrene-18C6 (CCDC $1045592 \dagger$ ) has been already reported. See ref. $25 b$.

32 M. Srebro-Hooper and J. Autschbach, Annu. Rev. Phys. Chem., 2017, 68, 399-420.

33 (a) S. Grimme, J. Antony, S. Ehrlich and H. Krieg, J. Chem. Phys., 2010, 132, 154104; (b) Y. Zhao and D. G. Truhlar, Theor. Chem. Acc., 2008, 120, 215-241.

34 With 18C4 and 16C4 derivatives, a less competitive solvent is necessary $\left(\mathrm{CH}_{2} \mathrm{Cl}_{2}\right.$ vs. $\left.\mathrm{CH}_{3} \mathrm{CN}\right)$ to compensate (possibly) for the loss of two oxygen atoms in comparison with 18C6 derivatives.

35 N. Berova, L. Di Bari and G. Pescitelli, Chem. Soc. Rev., 2007, 36, 914-931.

36 For the initial description of pyrene-based compounds exhibiting excimer associated CPL emission, see: (a) H. Brittain, D. L. Ambrozich, M. Saburi and J. H. Fendler, J. Am. Chem. Soc., 1980, 102, 6372-6374; (b) K. Kano, H. Matsumoto, S. Hashimoto, M. Sisido and Y. Imanishi, $J$. Am. Chem. Soc., 1985, 107, 6117-6118; (c) Y. Inai, M. Sisido and Y. Imanishi, J. Phys. Chem., 1990, 94, 2734-2735.

37 For other recent examples of CPL active pyrene-based platforms, see: (a) K. Takaishi, R. Takehana and T. Ema, Chem. Commun., 2018, 54, 1449-1452; (b) Y. Mimura, T. Nishikawa, R. Fuchino, S. Nakai, N. Tajima, M. Kitamatsu, M. Fujiki and Y. Imai, Org. Biomol. Chem., 2017, 15, 4548-4553; (c) J. Li, C. Yang, C. Huang, Y. Wan and W.-Y. Lai, Tetrahedron Lett., 2016, 57, 1256-1260; (d) S. Nakanishi, K. Nakabayashi, T. Mizusawa, N. Suzuki, S. Guo, M. Fujiki and Y. Imai, RSC Adv., 2016, 6, 9917299176; (e) M. Nakamura, J. Suzuki, F. Ota, T. Takada, K. Akagi and K. Yamana, Chem.-Eur. J., 2016, 22, 91219124; (f) T. Nishikawa, S. Kitamura, M. Kitamatsu, M. Fujiki and Y. Imai, ChemistrySelect, 2016, 4, 831-835; $(g)$ T. Nishikawa, N. Tajima, M. Kitamatsu, M. Fujiki and Y. Imai, Org. Biomol. Chem., 2015, 13, 11426-11431; (h) T. Amako, K. Nakabayashi, N. Suzuki, S. Guo, N. A. A. Rahim, T. Harada, M. Fujiki and Y. Imai, Chem. Commun., 2015, 51, 8237-8240; (i) K. Nakabayashi, T. Amako, N. Tajima, M. Fujiki and Y. Imai, Chem. Commun., 2014, 50, 13228-13230; (j) M. Inouye, K. Hayashi, Y. Yonenaga, T. Itou, K. Fujimoto, T. a. Uchida, M. Iwamura and K. Nozaki, Angew. Chem., Int. Ed., 2014, 53, 14392-14396.

38 E. M. Sánchez-Carnerero, A. R. Agarrabeitia, F. Moreno, B. L. Maroto, G. Muller, M. J. Ortiz and S. de la Moya, Chem.-Eur. J., 2015, 21, 13488-13500.

39 (a) K. Takaishi, R. Takehana and T. Ema, Chem. Commun., 2018, 54, 1449-1452; (b) Y. Mimura, T. Nishikawa, R. Fuchino, S. Nakai, N. Tajima, M. Kitamatsu, M. Fujiki and Y. Imai, Org. Biomol. Chem., 2017, 15, 4548-4553; (c) J. Li, C. Yang, C. Huang, Y. Wan and W.-Y. Lai, Tetrahedron Lett., 2016, 57, 1256-1260; (d) S. Nakanishi, K. Nakabayashi, T. Mizusawa, N. Suzuki, S. Guo, M. Fujiki 
and Y. Imai, $R S C$ Adv., 2016, 6, 99172-99176; (e) M. Nakamura, J. Suzuki, F. Ota, T. Takada, K. Akagi and K. Yamana, Chem.-Eur. J., 2016, 22, 9121-9124; (f) T. Nishikawa, S. Kitamura, M. Kitamatsu, M. Fujiki and Y. Imai, ChemistrySelect, 2016, 4, 831-835; $(g)$ T. Nishikawa, N. Tajima, M. Kitamatsu, M. Fujiki and Y. Imai, Org. Biomol. Chem., 2015, 13, 11426-11431; (h) T. Amako, K. Nakabayashi, N. Suzuki, S. Guo, N. A. A. Rahim, T. Harada, M. Fujiki and Y. Imai, Chem. Commun., 2015, 51, 8237-8240; (i) K. Nakabayashi, T. Amako, N. Tajima, M. Fujiki and Y. Imai, Chem. Commun., 2014, 50, 1322813230; (j) M. Inouye, K. Hayashi, Y. Yonenaga, T. Itou, K. Fujimoto, T. a. Uchida, M. Iwamura and K. Nozaki, Angew. Chem., Int. Ed., 2014, 53, 14392-14396; (k) Y. Inai, M. Sisido and Y. Imanishi, J. Phys. Chem., 1990, 94, 27342735; (l) K. Kano, H. Matsumoto, S. Hashimoto, M. Sisido and Y. Imanishi, J. Am. Chem. Soc., 1985, 107, 6117-6118; (m) H. Brittain, D. L. Ambrozich, M. Saburi and J. H. Fendler, J. Am. Chem. Soc., 1980, 102, 6372-6374.
40 Y. Sheng, J. Ma, S. Liu, Y. Wang, C. Zhu and Y. Cheng, Chem.-Eur. J., 2016, 22, 9519-9522.

41 F. Salerno, J. A. Berrocal, A. T. Haedler, F. Zinna, E. W. Meijer and L. Di Bari, J. Mater. Chem. C, 2017, 5, 3609-3615.

42 (a) J. Kumar, T. Nakashima, H. Tsumatori and T. Kawai, J. Phys. Chem. Lett., 2014, 5, 316-321; (b) J. Kumar, T. Nakashima, H. Tsumatori, M. Mori, M. Naito and T. Kawai, Chem.-Eur. J., 2013, 19, 14090-14097; (c) H. Tsumatori, T. Nakashima and T. Kawai, Org. Lett., 2010, 12, 2362-2365.

43 A $g_{\text {lum }}$ in the order of $10^{-4}$ is observed for the CPL signal associated with the monomer emission.

44 H. Tanaka, Y. Inoue and T. Mori, ChemPhotoChem, 2018, 2, 386-402.

45 S. Sinn, F. Biedermann, M. Vishe, A. Aliprandi, C. Besnard, J. Lacour and L. De Cola, ChemPhysChem, 2016, 17, 18291834. 